\title{
ESTABILIZAÇÃO UMERORRADIAL COM PARAFUSOS E POLIÉSTER TRANÇADO EM CÃO COM LUXAÇÃO TRAUMÁTICA DE COTOVELO
}

\author{
HUMERORADIAL STABILIZATION WITH SCREWS AND BRAIDED \\ POLYESTER IN DOG WITH TRAUMATIC ELBOW LUXATION
}

Leonardo Martins Leal ${ }^{1}$, Carla Nazaré Magalhães Parra ${ }^{2 *}$, Gabriela Maria Benedetti Vasques ${ }^{1}$, Diécila Milena Terezan ${ }^{1}$, Thalissa Fernanda Ciboldi ${ }^{3}$, Danielli Aparecida Lavelli ${ }^{1}$

${ }^{1}$ Centro Universitário Ingá, Maringá, PR, Brasil.

${ }^{2}$ Universidade Estadual de Maringá, Umuarama, PR, Brasil.

${ }^{3}$ Universidade Estadual do Norte do Paraná, Jacarezinho, PR, Brasil.

*Autor de correspondência: carlamagalhaesvet@hotmail.com

Recebido: 12 de março de 2020; Aceito: 18 de agosto de 2020.

\section{RESUMO}

Objetivou-se descrever o caso de um cão com luxação traumática de cotovelo que obteve bons resultados após correção ortopédica com parafusos e fio de poliéster trançado. $O$ tutor referiu que paciente se evadiu de sua residência e retornou após três dias sem apoiar o membro torácico esquerdo. O paciente foi encaminhado à Clínica Veterinária Uningá após 12 dias de seu retorno. Na avaliação clínica pôde-se observar: claudicação do membro torácico esquerdo, dor, crepitação e instabilidade da articulação do cotovelo com deslocamento lateral da cabeça do rádio e do olécrano em relação ao úmero distal. Confirmouse o diagnóstico de luxação lateral do cotovelo pelo exame radiográfico. 0 paciente foi submetido à cirurgia ortopédica e, após 15 dias do procedimento cirúrgico, observou-se boa reparação da ferida com discreta claudicação do membro operado. Após 90 dias de pós-operatório o paciente apresentava boa deambulação, sem indícios clínicos de claudicação, dor, instabilidade e crepitação do membro operado. Concluiu-se então com este relato que o uso dos parafusos associados ao fio de poliéster trançado em figura de " 8 " pode ser boa opção no tratamento da luxação traumática de cotovelo.

Palavras-chave: Articulação. Artrodese. Deslocamento. Ortopedia.

\section{ABSTRACT}

The objective was to describe the case of a dog with traumatic elbow luxation that obtained good results after orthopedic correction with screws and braided polyester in the form of an "8". The owner reported that the animal escaped from his home and returned after 3 days, without supporting the left thoracic limb. The patient was referred to the Uningá Veterinary Clinic 12 days after its return. In the clinical evaluation, it was possible to observe lameness without the support of the left thoracic limb, pain, crackling and instability of the elbow joint with lateral displacement of the radial head and olecranon in relation to the distal humerus. The diagnosis of lateral elbow luxation was confirmed by radiographic 
examination. The patient underwent orthopedic surgery and return 15 days after the surgical procedure with good wound healing and slight limping of the operated limb. After 90 days postoperatively, the patient had good walking, without clinical signs of lameness, pain, instability, and crackling of the operated limb. It was concluded with this report that the use of screws associated with the polyester braided in a form of an "8" can be a good option in the treatment of traumatic elbow dislocation.

Keywords: Arthrodesis. Displacement. Joint. Orthopedics.

\section{INTRODUÇÃO}

O cotovelo do cão é uma articulação complexa, sendo umas das articulações mais consistentes e estáveis do corpo. A articulação do cotovelo é formada pela junção úmero distal com o rádio e a ulna proximal, e é caracterizada como junção uniaxial por realizar somente o movimento de flexão e extensão (LIEBICH et al., 2016). Os ligamentos de maior relevância são os colaterais, medial e lateral; todavia, existem os ligamentos do olécrano oblíquo e anular do rádio (Figura 1). O cotovelo possui as características clássicas das articulações sinoviais como: ligamentos, líquido sinovial, cápsula articular, cavidade articular, cartilagem articular. O movimento do cotovelo é tipo dobradiça, sendo responsável pela maior parte das forças que o membro realiza (DYCE; SACK; WENSING, 2010; LAHUNTA; EVANS, 2013).

A luxação traumática do cotovelo, ou deslocamento do cotovelo, está geralmente associada a um trauma brusco nesta articulação, causando consequentemente o deslocamento lateral do rádio e da ulna em relação ao úmero (SCHULTZ, 2013). A luxação lateral do cotovelo é mais comum do que a luxação medial, pois o côndilo medial do úmero, por ser maior, evita o deslocamento do rádio e da ulna nesta direção. Assim, as luxações mediais tornam-se mais raras (RAHAL et al., 2000; SCHULZ, 2013).

A luxação umeroradioulnar pode ser traumática ou congênita. Dentre as traumáticas, a luxação pode ser imediata, especialmente em pacientes atropelados por veículos automotivos, ou como consequência de fraturas fisárias maltratadas de rádio e ulna, as quais podem levar ao fechamento prematuro das linhas de crescimento, ocasionando incongruência rádioulnar, o que favorece as luxações (DENNY; BUTTERWORTH, 2006). A luxação congênita, por sua vez, possui etiologia até o momento desconhecida (SCHULZ, 2013).

O diagnóstico da luxação do cotovelo é feito pelos achados clínicos e confirmados pelos exames de imagem: radiografia, tomografia computadorizada (TC) e ressonância magnética (RM) (PIERMATTEI; FLO; DECAMP, 2009). O paciente apresenta claudicação e o membro torácico fica flexionado. Na luxação lateral, a cabeça do rádio e o olécrano ficam deslocados lateralmente em relação ao úmero, o côndilo lateral do úmero por sua vez fica oculto à palpação. Radiografias simples identificam facilmente a luxação da articulação umeroradioulnar (PIERMATTEI; FLO; DECAMP, 2009; SCHULZ, 2013).

O tratamento pode ser conservador ou cirúrgico. No tratamento conservador, a redução fechada da articulação é possível, todavia os melhores resultados são obtidos quando realizada nos primeiros dias após a lesão, na qual 
há mínima contração muscular (PIERMATTEI; FLO; DECAMP, 2009). Caso a redução seja obtida, aplica-se bandagem, tala ou gesso com o membro torácico estendido para manter a estabilidade dessa articulação, até que o processo cicatricial ocorra (SCHULZ, 2013).

O tratamento cirúrgico consiste na redução aberta da luxação e consequente estabilização da articulação do cotovelo. A cirurgia é indicada quando não se obtém sucesso na redução fechada (TOMLISON, 2008; SCHULZ, 2013). Foram descritas diversas técnicas cirúrgicas para corrigir as luxações de cotovelo, todas necessitam de implantes ortopédicos para a estabilização da articulação. Independente da técnica adotada, todas podem apresentar complicações como reluxação, infecções iatrogênicas, migração ou quebra dos implantes, entre outras. O prognóstico é bom quando a luxação é reduzida e estabilizada até sete dias após o trauma (SCHULZ, 2013). Ante ao exposto, e devido à alta incidência de injúrias ortopédicas ocasionadas por acidentes automotivos envolvendo os cães, bem como pela escassez de trabalhos sobre luxação lateral traumática na literatura nacional, objetivou-se descrever o caso de uma cadela com luxação lateral traumática crônica de cotovelo que obteve sucesso após correção cirúrgica por meio da estabilização umerorradial, com parafusos e fio de poliéster trançado em figura de "8'.

\section{CASO CLÍNICO}

Foi atendida, na Clínica Veterinária Uningá, de Maringá-PR, uma fêmea, da espécie canina, sem raça definida de 8 anos, $20 \mathrm{~kg}$, com claudicação do membro torácico esquerdo. O proprietário relatou que a paciente fugiu de casa e retornou após três dias com claudicação.

A paciente chegou à clínica veterinária após 15 dias do incidente. No exame físico geral, não verificaram alterações dignas de nota. No exame ortopédico, notou-se dor, crepitação e instabilidade da articulação do cotovelo esquerdo. Na palpação, verificou-se deslocamento lateral da cabeça do rádio e do olecrano em relação ao úmero distal. O hemograma e as enzimas séricas alanina aminotransferase e creatinina apresentavam valores dentro dos padrões de normalidade. O diagnóstico de luxação traumática de cotovelo foi confirmado pelo exame radiográfico do membro torácico esquerdo, nas posições mediolateral (M) e craniocaudal (L) (Figura 1).

Após o diagnóstico da luxação, indicou-se a cirurgia para a sua correção. A paciente recebeu pré-anestesia com acepromazina $(0,05 \mathrm{mg} / \mathrm{Kg})$ associada à morfina $(0,5 \mathrm{mg} / \mathrm{kg})$ pela via intramuscular. A indução anestésica foi realizada com propofol $(10 \mathrm{mg} / \mathrm{Kg})$, cetamina $(1 \mathrm{mg} / \mathrm{kg})$ e midazolan $(0,2 \mathrm{mg} / \mathrm{kg})$ pela via intravenosa e a manutenção anestésica foi feita com isofluorano.

Realizou-se tricotomia ampla da região do cotovelo esquerdo, colocou-se a paciente em decúbito lateral direito e realizou-se a antissepsia prévia e definitiva com solução clorexidine $2 \%$ degermante, seguida por álcool $70 \%$. Antes da primeira incisão, foi feito o bloqueio do plexo braquial na região do vazio torácico, com lidocaína a $2 \%(4 \mathrm{mg} / \mathrm{Kg})$. 
Figura 1 - Imagens Radiográficas do Cotovelo Esquerdo de cão.

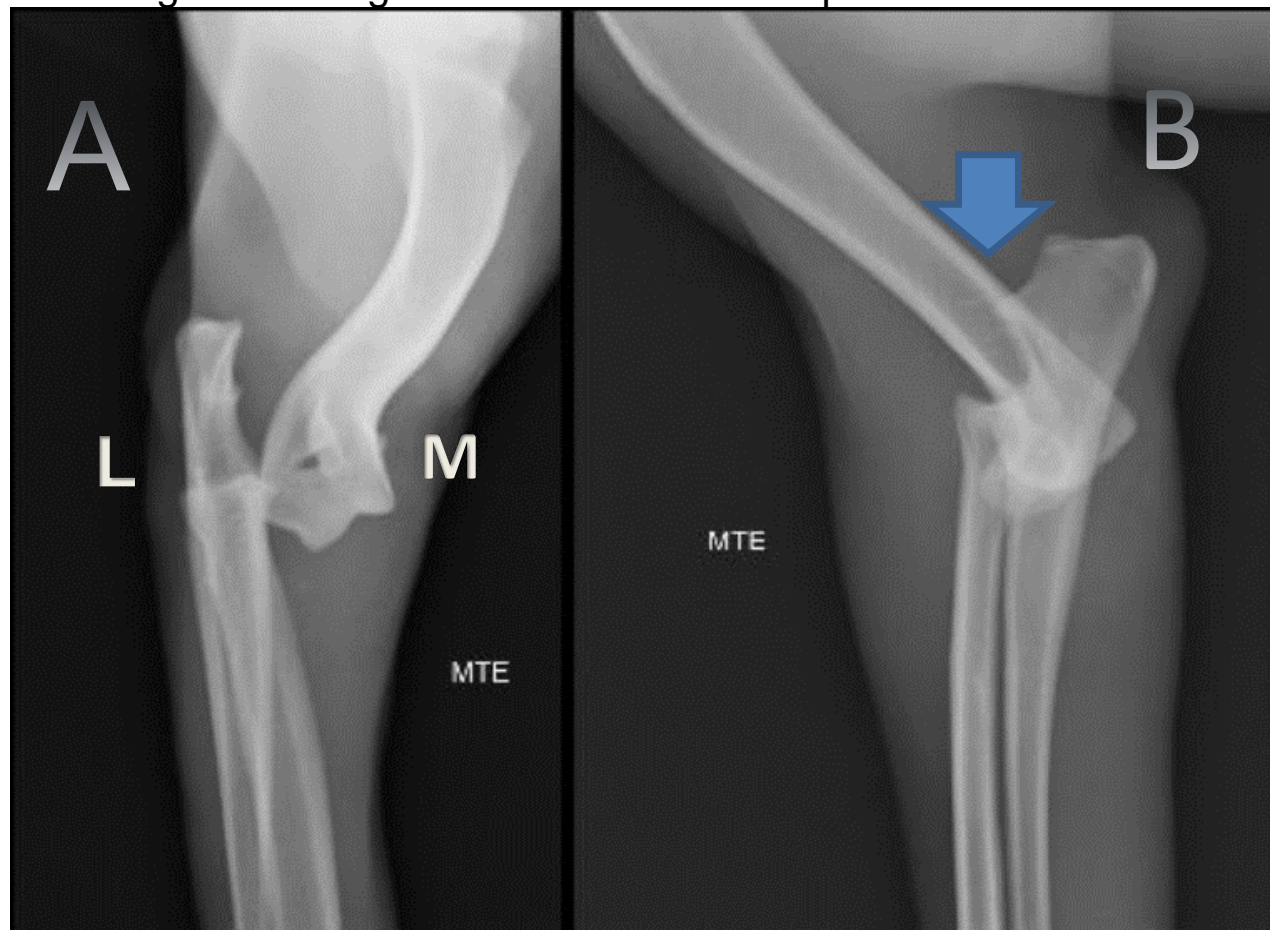

Fonte: Os autores.

Notas: Em A, imagem crânio caudal: observa-se deslocamento lateral do rádio em relação ao úmero esquerdo. $\mathrm{Em} \mathrm{B}$, imagem médio lateral: observa-se úmero distal sobreposto por rádio, sendo que a incisura troclear (seta) está livre, evidenciando a luxação da articulação umerorradial.

A incisão cutânea do cotovelo foi feita na região lateral do cotovelo esquerdo, estendendo-se do terço médio do úmero, até o terço médio do rádio. Incisou-se as fáscias, os tecidos oriundos da fibrose decorrente da cronicidade do quadro e a cápsula articular, que permitiu a identificação da cabeça do rádio e o epicôndilo lateral do úmero. Com auxílio de um afastador de Homman, o rádio e a ulna foram tracionados em direção aos côndilos umerais para facilitar a redução da luxação. Após a redução, um parafuso de $2,7 \mathrm{~mm} \times 25 \mathrm{~mm}$ foi fixado na cabeça do rádio e outro de $2,7 \mathrm{~mm} \times 23 \mathrm{~mm}$ no côndilo lateral do úmero. Para estabilizar a articulação, mimetizando a função do ligamento colateral lateral, aplicou-se uma sutura em figura de "8" ao redor dos parafusos com fio de poliéster trançado número 5 . A cápsula articular e as fáscias foram aproximadas com fio de náilon 2-0 em padrão simples contínuo. O tecido subcutâneo foi aproximado com poliglecaprone 25 3-0 e a pele foi suturada em padrão Wolff com fio de náilon 3-0. Radiografias pós-operatórias foram realizadas (Figura 2).

Realizou-se tala em Spica que permaneceu até a retirada dos pontos, aos 15 dias de pós-operatório. Foram prescritos, por via oral, Ranitidina $(2,2 \mathrm{mg} / \mathrm{Kg}$, BID, 15 dias) Cloridrato de Tramadol (3mg/Kg, BID, 5 dias), Dipirona $(25 \mathrm{mg} / \mathrm{Kg}$, BID, 5 dias) e Cefalexina $(30 \mathrm{mg} / \mathrm{Kg}$, BID, 10 dias) como medicações pósoperatórias.

Com 15 dias de pós-operatório, o paciente retornou ao hospital para retirada da tala e os pontos. A ferida estava cicatrizada e a paciente apresentava discreta claudicação com apoio do membro operado. Após 30 dias da cirurgia, a 
paciente retornou com boa locomoção, sem indícios clínicos de claudicação. Após 90 dias a paciente foi novamente reavaliada e apresentava boa deambulação, sem indícios clínicos de claudicação, dor, instabilidade e crepitação do membro operado. O proprietário descreveu a locomoção da cadela como "normal".

Figura 2: Imagens radiográficas pós operatórias.

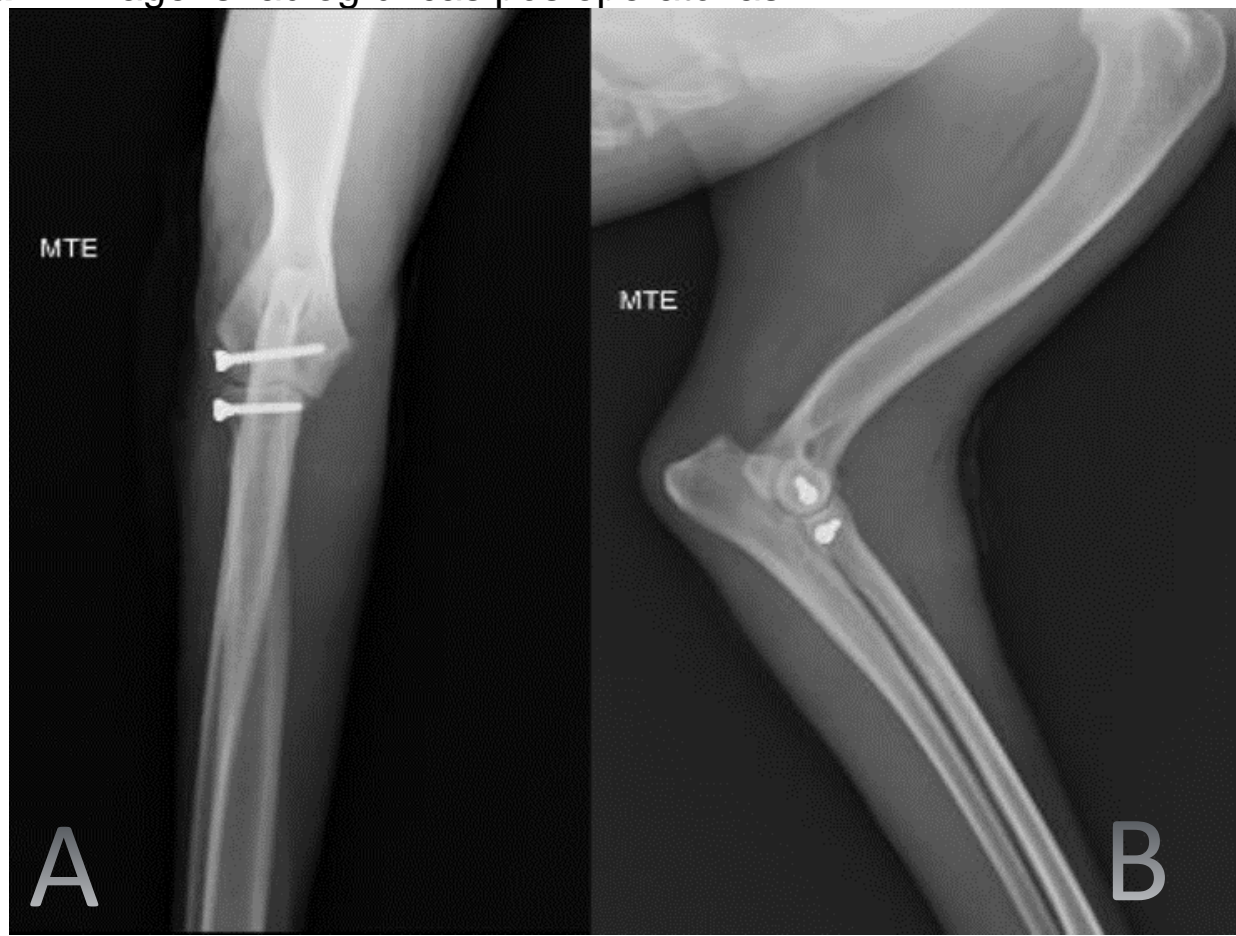

Fonte: os autores.

Notas: Em A, imagem crânio caudal, e B, imagem médio lateral: Observam-se a presença de dois parafusos, um em extremidade distal de úmero e outro em extremidade proximal de rádio.

\section{DISCUSSÃO}

A luxação de cotovelo em cães acomete, especialmente, raças de pequeno e médio porte (MCDONNEL, 2004) como no caso em questão. Porém, os machos apresentam maior incidência (RAHAL et al., 2000), diferente do observado no presente relato, o qual trata-se de uma fêmea.

Como observado neste caso, a luxação lateral é mais comum que a medial, devido à anatomia do cotovelo dos cães, que possuem o côndilo medial do úmero maior, evitando assim o deslocamento para essa direção (SCHULZ, 2013).

O diagnóstico de luxação traumática de cotovelo é frequentemente realizado com base no exame físico e radiografias. Os exames de imagem permitem ainda verificar possíveis fraturas associadas, além de sugerir lesões ligamentares pelo deslocamento apresentado entre os ossos que compõem essa articulação (PIERMATTEI; FLO; DECAMP, 2009). Nessa paciente, a radiografia simples confirmou o diagnóstico de luxação lateral de cotovelo e pelo 
deslocamento observado na imagem craniocaudal, suspeitou-se de ruptura dos ligamentos colaterais.

Embora a redução fechada seja opção para o tratamento da luxação lateral do cotovelo, ela apresenta melhores resultados quando executada nos primeiros dias após a lesão (SCHULZ, 2013), fato este que impossibilitou a realização desta manobra no paciente do presente relato, uma vez que a luxação era crônica. Assim, a redução aberta foi prontamente indicada.

Para reduzir a luxação que era crônica, foi necessário o uso de um afastador de Homman para deslocar o rádio e a ulna contra o côndilo lateral do úmero. Esta manobra, porém, é desaconselhada, pois pode causar lesões na cartilagem articular (PIERMATTEI; FLO; DECAMP, 2009), favorecendo o desenvolvimento de doença articular degenerativa (DAD) (SCHULZ, 2013). Todavia, pela cronicidade e dificuldade de alinhamento da articulação ela se fez necessária e até o período de avaliação pós-operatório, a paciente não apresentava sinais clínicos ou de imagem sugestiva de DAD.

Ainda quanto ao uso de implantes, na redução aberta, pode-se mimetizar a função do ligamento colateral lateral com o uso de dois parafusos e um fio de aço em forma de "8". Para esta técnica foram descritos bons resultados, porém uma complicação comum é a quebra ou afrouxamento do fio (DENNY; BUTTERWORTH, 2006). Assim, nesse caso, optou-se, alternativamente, pelo uso do fio de poliéster trançado, que possui boa pressão de apoio e poucas complicações tardias relacionadas à quebra do fio como relatado por (PRADA et al., 2011) em seu estudo na avaliação do fio de poliéster na correção da ruptura do ligamento cruzado cranial de cães.

Dentre as possíveis complicações cirúrgicas, são descritas: reluxação, redução da amplitude do cotovelo, infecção iatrogênica, migração de implantes, irritação dos tecidos moles adjacentes e DAD (SCHULZ, 2013). No estudo retrospectivo de Sajik et al. (2016), foram analisados 37 cães com luxação de cotovelo traumática; o autor refere a reluxação (27\%) como a maior complicação pós-operatória. No caso em questão, até a última avaliação da paciente, não se presenciou nenhuma dessas intercorrências.

No pós-operatório de reduções abertas é indicado o uso de tala flexível por 5 a 7 dias (PIERMATTEI; FLO; DECAMP, 2009; SCHULZ, 2013). Nessa paciente do presente relato, fez-se o uso da tala por mais dias, pois a lesão era crônica, e a contratura muscular bem significativa, o que poderia ter ocasionado maior estresse e falha dos implantes caso a tala fosse retirada num curto espaço de tempo.

Após a retirada da bandagem são indicados exercícios de flexão e extensão (PIERMATTEI; FLO; DECAMP, 2009), visto que a fisioterapia pósoperatória proporciona reabilitação mais rápida do membro (MUZZI; REZENDE; MUZZI, 2009). Todavia, a paciente retornou à ambulação com boa locomoção/deambulação logo após a retirada da tala, o que fez com que a fisioterapia fosse dispensada. 


\section{CONCLUSÃO}

Conclui-se com o relato em questão, que a correção cirúrgica de luxação lateral traumática de cotovelo com o uso de parafusos e fio poliéster trançado em figura de "8", pode ser boa opção cirúrgica para esta afecção.

\section{REFERÊNCIAS}

BEHRENS, F.; JOHNSON, W. Unilateral external fixation methods to increase and reduce frame stiffness. Clinical Orthopaedics and Related Research, v. 241, p. 48-56, 1989.

BRIENZA, P. D.; JUNIOR, A. C. C. L. Ressonância Magnética na avaliação da articulação do joelho em cães. 2013. Disponível em: < http://docplayer.com.br/9501971-Xxii-congresso-de-pos-graduacao-da-

ufla.html>. Acesso em 04 de julho de 2016.

DENNY, H. R.; BUTTERWORTH, J. S. Cirurgia ortopédica em cães e gatos. 4. ed. São Paulo: Roca, 2006. 504p.

DYCE, K. M.; SACK, W. O.; WENSING, C. J. G. Tratado de Anatomia Veterinária. 4. ed. Rio de Janeiro: Elsevier, 2010. 872p.

HAMILTON, K. et al. Caudal elbow luxation in a dog managed by temporaray transarticular external skeletal fixation. Hindawi, v. 2014, p. 1-7, 2014.

LAHUNTA, E. D.; EVANS, H. E. Miller's anatomy of the dog. 4. ed. Philadelphia: Elsevier, 2013. 872p.

LIBICH, H. G. et al. Esqueleto do membro torácico. In: KÖNIG, H. E.; LIEBICH, H. Anatomia dos Animais Domésticos. 6. ed. Porto Alegre: Artmed, 2016. $187 p$.

MARIANI, O. M. et al. Estabilização temporária bilateral para a correção de luxação de cotovelo. Revista Investigação, v. 15, n. 1, p. 90-93, 2016.

MCDONEL H. L. Unilateral congenital elbow luxation in a Cavalier King Charles Spaniel. The Canadian Veterinary Journal, v. 45, n. 11, p. 941-943, 2004.

MITCHELL, K. E. Traumatic elbow luxation in 14 dogs and 11 cats. Australian Veterinary Journal, v. 89, n. 6, p. 213-216, 2011.

MUZZI, L. A. L.; REZENDE, C. M. F.; MUZZI, R. A. L. Fisioterapia após substituição artroscópica do ligamento cruzado cranial em cães: I - avaliação clínica, radiográfica e ultrassonográfica. Arquivo Brasileiro de Medicina Veterinária e Zootecnia, v. 61 n. 4, p. 805-814, 2009. 
PENWICK, R. C. Arthrodesis. Veterinary Clinics of North America: Small Animal Practice, PubMed, v. 17, n. 4, p. 821-840, 1987.

PIERMATTEI, D. L.; FLO, G.; DECAMP, C. E. Ortopedia e tratamento de fraturas em pequenos animais. 4. ed. Barueri: Manole, 2009. 896p.

PRADA, T. C. et al. Estudo comparativo entre uso de fio de poliamida $x$ fio de poliéster intra-articular para o tratamento de ruptura do ligamento cruzado cranial em cães. Revista de Educação Continuada em Medicina Veterinária e Zootecnia, v. 9, n. 2, p. 35-36, 2011.

RAHAL, S. C. et al. Reduction of humeroulnar congenital elbow luxation in 8 dogs by using the transarticular pin. The Canadian Veterinary Journal. v. 41, n.11, p. 849-853, 2000.

RYCKE, L. M. D. et al. Computed tomography of the elbow joint in clinically normal dogs. American Journal of Veterinary Research. v. 63, n. 10 p. 14001407, 2002.

SAJIK, D. et al. Multi-centre retrospective study of long-term outcomes following traumatic elbow luxation in 37 dogs. Journal of Small Animal Practice. v. 57, n. 8, p.422-428, 2016.

SCHULZ, K. S. Afecções articulares. In: FOSSUM, T. W. Cirurgia de Pequenos Animais. 4. ed. Rio de janeiro: Elsevier, 2013. p.1215-1374.

TIDWELL, A. S. Princípios da tomografia computadorizada e da imagem por ressonância Magnética. In: THRALL, D.E. Diagnóstico de Radiologia Veterinária. 5. ed. Rio de janeiro: Elsevier, 2010. p.50-77.

TOMLINSON, J. Fraturas e deformidades de crescimento de rádio e ulna e luxação de cotovelo. In: BIRCHARD, S. J.; SHERDING, R. G.; Manual Saunders - Clínica de Pequenos Animais. 3. ed. São Paulo: Roca, 2008. p.1106-1116. 